\title{
Treatment of Cirrhosis-Associated Hyponatremia Refractory to Vasopressin 2-Receptor Antagonist
}

\author{
Ajay Yadlapati ${ }^{\mathrm{a}}$, Arksarapuk Jittirat ${ }^{\mathrm{b}}$, Minhtri K. Nguyen ${ }^{\mathrm{a}, \mathrm{c}}$
}

\begin{abstract}
Hyponatremia in patients with cirrhosis is due to impaired water excretion from persistent release of antidiuretic hormone induced by diminished effective circulatory volume (ECV). The treatment of cirrhosis-associated hyponatremia is free water restriction and vasopressin-2-receptor antagonists (V2RAs). We report a cirrhotic patient with hyponatremia who failed to improve with V2RAs but corrected with treatment of hepatorenal syndrome (HRS). The failure of hyponatremia to improve with V2RAs was likely due to avid renal proximal $\mathrm{Na}^{+}$and $\mathrm{H}_{2} \mathrm{O}$ reabsorption from decreased $\mathrm{ECV}$, resulting in diminished fluid delivery to the collecting tubule. Midodrine and octreotide improved the systemic hemodynamics and increased distal fluid delivery resulting in resolution of hyponatremia. To date, this is the first case report of a cirrhotic patient with hyponatremia refractory to V2RAs that resolved with treatment of HRS.
\end{abstract}

Keywords: Hyponatremia; Cirrhosis; Vasopressin receptor antagonist

\section{Introduction}

Hyponatremia in cirrhotic patients was described for the first time in 1950 by Eisenmenger et al [1]. It is a common complication in patients with advanced liver disease, $49.4 \%$ of patients with cirrhosis have serum sodium concentration less than $135 \mathrm{mmol} / \mathrm{L}$. Hyponatremia in the setting of cirrhosis

\footnotetext{
Manuscript accepted for publication March 11, 2014

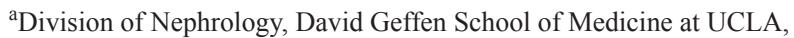
Los Angeles, CA, USA

${ }^{\mathrm{b}}$ Division of Nephrology, UCLA-Olive View Medical Center, Los Angeles, CA, USA

${ }^{\mathrm{c}}$ Correspondence to: Minhtri K. Nguyen, Ronald Reagan UCLA Medical Center, 757 Westwood Blvd, Rm. 7501B, Los Angeles, CA 90095-7417, USA. Email: mtnguyen@mednet.ucla.edu

doi: http://dx.doi.org/10.14740/jmc1745w
}

is associated with severe ascites, high frequency of hepatic encephalopathy, spontaneous bacterial peritonitis and hepatorenal syndrome (HRS) [2]. Additionally, hyponatremia is also a powerful predictor of morbidity and mortality in patients with cirrhosis.

The etiology of hyponatremia in cirrhotic patients can be attributed to either intravascular volume depletion commonly found in patients who receive high dose of diuretics or hypervolemic hyponatremia due to diminished effective circulatory volume (ECV) resulting from splanchnic vasodilatation. The diminished ECV due to splanchnic vasodilatation will lead to increased secretion of antidiuretic hormone $(\mathrm{ADH})$, which in turn results in decreased ability to excrete the daily water intake. Whereas the treatment of hypovolemic hyponatremia secondary to overdiuresis is to discontinue the diuretics and to restore the intravascular volume depletion with isotonic saline, the mainstay of treatment for hypervolemic hyponatremia secondary to the impaired water excretion is free water restriction. Recently, vasopressin-2receptor antagonist (V2RA) has been used as an alternative treatment in cirrhotic patients whose hyponatremia fails to correct with free water restriction alone [3]. The increase in serum sodium concentration occurs within the first few days of treatment and ranges from 2 to $7 \mathrm{mmol} / \mathrm{L}$ on average [4]. To date, there has been no report of the failure of V2RAs in correcting hyponatremia in patients with cirrhosis. We report a patient with cirrhosis whose hyponatremia failed to improve with tolvaptan and conivaptan but subsequently corrected with treatment with midodrine and octreotide.

\section{Case Report}

A 51-year-old female with past medical history significant for alcoholic cirrhosis, chronic obstructive pulmonary disease and multiple admissions for hyponatremia was admitted for management of severe hyponatremia. On presentation, the patient had a serum sodium concentration of 118 $\mathrm{mmol} / \mathrm{L}$ associated with a serum and urine osmolality of 254 and $519 \mathrm{mOsm} / \mathrm{kg} \mathrm{H}_{2} \mathrm{O}$ respectively. The patient's hyponatremia failed to improve with free water restriction. Therefore, tolvaptan, a selective V2RA, was started with close 


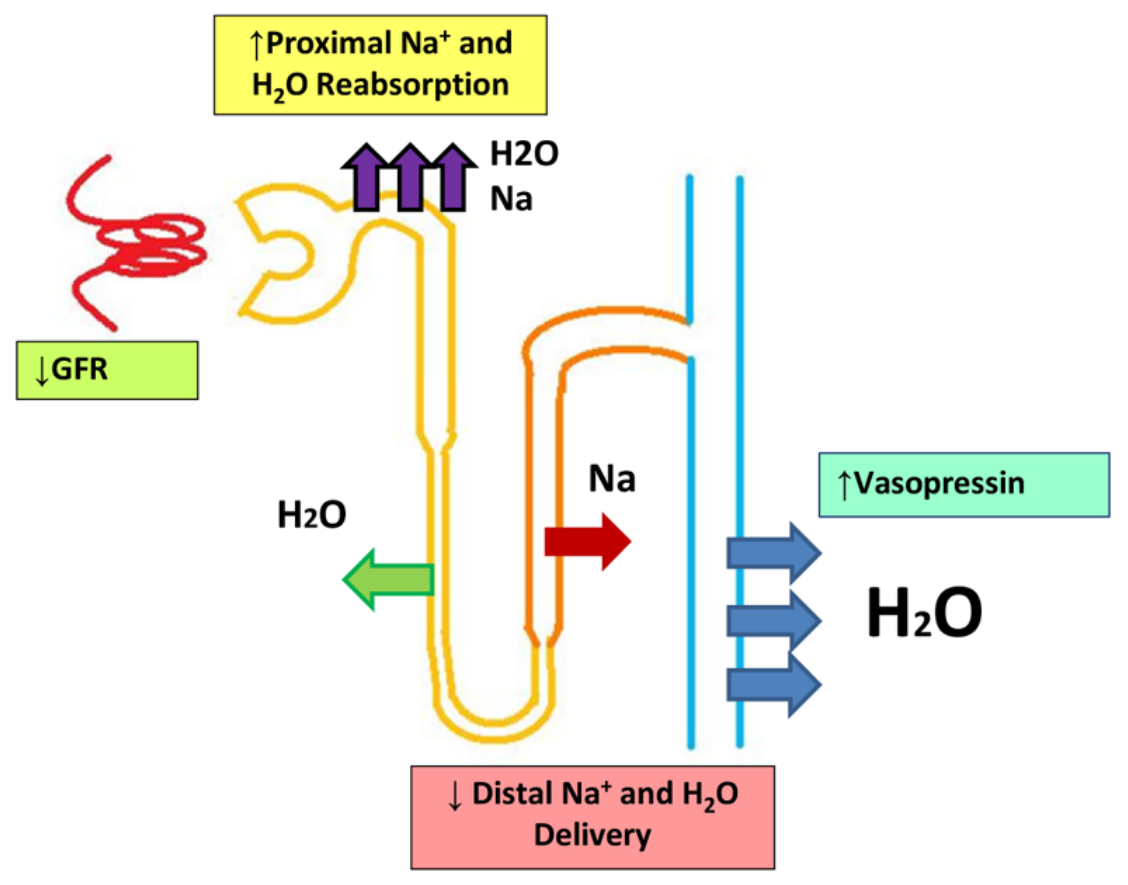

Figure 1. Impaired urinary dilution in cirrhosis.

monitoring of the patient's serum sodium concentration (Of note, tolvaptan was FDA approved for treatment of hyponatremia secondary to cirrhosis at that time). Despite adequate up-titration from 15 to $60 \mathrm{mg}$ over a 96-h period, her serum sodium level remained relatively unchanged at a level of 120 $\mathrm{mmol} / \mathrm{L}$. Due to the potential concern for inadequate gastrointestinal absorption of the drug, a decision was made to change from oral tolvaptan to intravenous conivaptan. Her serum sodium concentration, however, minimally improved to $123 \mathrm{mmol} / \mathrm{L}$ even after up-titration of the conivaptan to the maximum dose. On hospital day 13 , the patient was started on midodrine and octreotide for treatment of acute kidney injury thought to be due to HRS, which slowly brought the patient's serum sodium level from 122 to $132 \mathrm{mmol} / \mathrm{L}$ over a 5 -day course coinciding with the resolution of the HRS. Repeat laboratory values revealed a stable serum sodium level of $135 \mathrm{mmol} / \mathrm{L} 2$ weeks after initiation of the drugs.

\section{Discussion}

Hyponatremia commonly occurs in patients with cirrhosis. In cirrhosis, the pathogenesis is thought to be related to the hemodynamic changes and secondary neurohumoral adaptation that occurs in these patients. Patients with cirrhosis usually have a marked reduction in systemic vascular resistance (SVR) and mean arterial pressure due to splanchnic vasodilatation. The splanchnic vasodilatation in cirrhotic patients results in arterial underfilling, and the resultant ECV depletion in these patients stimulates the secretion of $\mathrm{ADH}$.
The elevated ADH secretion leads to increased water reabsorption in the collecting tubule, which in turn results in decreased ability to excrete the daily water intake. Therefore, V2RAs can be an effective therapy for cirrhosis-associated hyponatremia by antagonizing the action of $\mathrm{ADH}$ at the collecting tubule. By reducing the urinary concentration in the collecting duct, V2RAs result in the urinary excretion of electrolyte-free water excretion.

In our patient, her hyponatremia, however, failed to improve with free water restriction and V2RAs. Her hyponatremia was refractory to tolvaptan and conivaptan despite a reduction in urine osmolality from $519 \mathrm{mOsm} / \mathrm{kg} \mathrm{H}_{2} \mathrm{O}$ to 242 $\mathrm{mOsm} / \mathrm{kg} \mathrm{H} \mathrm{H}_{2} \mathrm{O}$. Her hyponatremia persisted until she was started on midodrine (selective alpha-1 adrenergic agonist) and octreotide (a somatostatin analog) for treatment of HRS. Treatment with midodrine and octreotide resulted in the resolution of the HRS as well as hyponatremia with an associated increment in her urinary output and relatively constant urinary osmolality.

The lack of response to the V2RAs in our patient was due to disruption in the mechanisms involved with dilute urine formation. The normal physiology of urinary dilution is determined by numerous factors such as normal glomerular filtration rate, adequate delivery of $\mathrm{H}_{2} \mathrm{O}$ to the diluting segments of the nephron mediated by inhibition of proximal $\mathrm{Na}^{+}$and $\mathrm{H}_{2} \mathrm{O}$ absorption, intact $\mathrm{Na}^{+}$and $\mathrm{Cl}^{-}$reabsorption at both the thick ascending limb of the loop of Henle and distal convoluted tubules, and the inhibition of $\mathrm{H}_{2} \mathrm{O}$ absorption at the collecting duct resulting from the suppression of $\mathrm{ADH}$ secretion. In light of the aforementioned factors, the efficacy 
of the V2RAs in cirrhosis will be limited by the decrease in distal delivery of the filtrate to the collecting tubule which is the site of ADH action (Fig. 1). Therefore, the lack of response to the V2RAs in our patient was likely due to the decreased GFR and avid proximal sodium and fluid reabsorption (Fig. 1). The low urinary $\mathrm{Na}^{+}$of $<20 \mathrm{mmol} / \mathrm{L}$ was consistent with the increased proximal sodium and fluid reabsorption resulting from the decreased ECV. This will in turn lead to diminished distal fluid delivery to the collecting tubule, the site of ADH action. Distal fluid delivery is best assessed by determining the sum of the fraction of filtered chloride excreted (chloride clearance/GFR) plus the fractional free water clearance (free water clearance/GFR) [5]. Unfortunately, urinary chloride concentration before and after treatment with midodrine and octreotide was not available to assess distal fluid delivery in this patient. However, the improvement in hyponatremia with midodrine and octreotide in our patient was likely due to increased distal fluid delivery to the collecting tubule as reflected in the increase in urinary output in the setting of relatively constant urinary osmolality. Urinary osmolality initially decreased from 519 $\mathrm{mOsm} / \mathrm{kg}$ to $242-250 \mathrm{mOsm} / \mathrm{kg}$ after the administration of tolvaptan and conivaptan. After treatment of HRS with midodrine and octreotide, urinary osmolality remained relatively constant at $245-248 \mathrm{mOsm} / \mathrm{kg}$. Although the urinary osmolality remained relatively constant, her urinary output increased from $570-1200 \mathrm{~mL}$ to $1750-1850 \mathrm{~mL}$, thereby resulting in an increment in free water clearance. Free water clearance increased from $0.076 \mathrm{~L} /$ day prior to initiation of midodrine and octreotide to $0.31 \mathrm{~L} /$ day after administration of midodrine and octreotide. Unfortunately, urinary sodium and potassium concentrations were not available to calculate urinary electrolyte-free water clearance. The increase in free water clearance was likely due to increased fluid delivery to the diluting segments of the nephron and collecting tubule due to improvement in the ECV induced by midodrine and octreotide.

To date, this is the first case report of a cirrhotic patient with hyponatremia refractory to V2RAs which resolved with treatment with midodrine and octreotide. Further studies are needed to help substantiate the effectiveness of this novel therapeutic approach in cirrhotic patients whose hyponatremia fails to improve with V2RAs. In these patients, assessment of distal fluid delivery will be helpful in elucidating the mechanism of action of midodrine and octreotide in the correction of hyponatremia refractory to V2RAs.

\section{Conflict of Interests}

The authors declare that they have no conflict of interest.

\section{References}

1. Eisenmenger WJ, Blondheim SH, Bongiovanni AM, Kunkel HG. Electrolyte studies on patients with cirrhosis of the liver. The Journal of clinical investigation. Nov 1950;29(11):1491-1499.

2. Angeli P, Wong F, Watson H, Gines P, Investigators C. Hyponatremia in cirrhosis: Results of a patient population survey. Hepatology. Dec 2006;44(6):1535-1542.

3. Gines P, Berl T, Bernardi M, et al. Hyponatremia in cirrhosis: from pathogenesis to treatment. Hepatology. Sep 1998;28(3):851-864.

4. Gines P, Guevara M. Hyponatremia in cirrhosis: pathogenesis, clinical significance, and management. Hepatology. Sep 2008;48(3):1002-1010.

5. Dusing R, Moritz J, Glanzer K, Kramer HJ. Effect of angiotensin II and captopril on renal tubular function in man. British journal of clinical pharmacology. Jan 1985;19(1):29-35. 